\title{
Physical stability of microliposomes in bene (Pistacia atlantica) oil with different formulations
}

\author{
Mahsa Nikkhah ${ }^{1}$, Zhaleh Khoshkhoo ${ }^{2 *}$, Seyed Ebrahim Hosseini ${ }^{3}$, Peyman Mahasti Shotorbani ${ }^{4}$, \\ Afshin Akhondzadeh Basti ${ }^{5}$ \\ ${ }^{1}$ Department of Food Science and Technology, North Tehran Branch, Islamic Azad University, Tehran, Iran; ${ }^{2}$ Faculty of \\ Food Science and Technology, Tehran North Branch, Islamic Azad University, Tehran, Iran; ${ }^{3}$ Department of Food Science \\ and Technology, Tehran Science and Research Branch, Islamic Azad University, Tehran, Iran; ${ }^{4}$ Department of Food \\ Quality Control and Hygiene, Science and Research Branch, Islamic Azad University, Tehran, Iran; ${ }^{5}$ Department of Food \\ Hygiene, Faculty of Veterinary Medicine, University of Tehran, Iran
}

*Corresponding Author: Zhaleh Khoshkhoo, Faculty of Food Science and Technology, Tehran North Branch, Islamic Azad University, Tehran, Iran. Email: Zh_khoshkhoo@iau-tnb.ac.ir

Received: 19 October 2020; Accepted: 18 November 2020; Published: 21 December 2020 (C) 2020 Codon Publications

\begin{abstract}
Bene oil (pistacia atlantica), as a plant source, is rich in phenolic and tocopherol compounds and has significant antioxidant, therapeutic and antimicrobial effects. Encapsulation of hydrophobic compounds in liposome system is an ideal solution for protecting them against destruction during storage. An important advantage of liposomes is the encapsulation of hydrophilic, hydrophobic and amphiphilic compounds by using natural phospholipids, such as lecithin, with beneficial effects. The aim of this study is to encapsulate the bene kernel oil in the form of microliposomes. For this purpose, the effect of composition of liposomes based on lecithin and cholesterol was studied using the Mozafari method. Liposomes are prepared using lecithin and cholesterol in the ratios of 60:0, 50:10, 40:20 and 30:30. Particle size, size distribution, zeta potential and encapsulation efficiency were characterised. According to the result, the size of liposomes was dependent on their composition, but the wasaffected significantly affected by adding cholesterol $(P<0.05)$. Average diameter of the particles was between 4 and 9 $\mu \mathrm{m}$. Liposome with a ratio of 40:20 had the smallest size. By applying cholesterol, zeta potential increased from $16.4 \mathrm{mV}$ to $32.39 \mathrm{mV}$, which indicates electrostatic stability of liposomes. In general, with encapsulation efficiency of $84.33 \%$, the ratio of $40: 20$ is considered as an ideal concentration in the formulation of microliposomes. Based on the results, bene oil-loaded liposomes with a lecithin:cholesterol formulation ratio of :as 40:20 was chosen as an optimal formulation because of its smaller particle size, higher zeta potential and suitable stability, which can be used in trapping, delivering and releasing hydrophilic, adipose-friendly and amphiphilic compounds (dual-friendly).
\end{abstract}

Keywords: cholesterol, encapsulate, lecithin, Mozafari method, phenolic compound, tocopherols

\section{Introduction}

Pistacia atlantica belongs to the Anacardiaceae family. Bene or wild pistachio is a native of Iran and found distributed in its Zagros region (Rezaie et al., 2015). Natives use bene as food and medicine (Farhoosh et al., 2008b). Bene fruit consists of kernel, wooden shell and green hull. Kernel is about $25 \%$ of whole bene fruit and is used to extract oil (Sharif et al., 2009). The oil content of kernel is about $55-58 \%$. Analysis of kernel oil reveals that it is a good source of monounsaturated fatty acid (MUFA) and the oil has significant amount of phenolic compounds and tocopherols, which are considered as natural antioxidants (Farhoosh et al., 2008b, Wong et al., 1988). 
Consumer preferences towards functional foods are increasing rapidly. Phenolic and tocopherols, which are secondary metabolites of plants, are bioactive compounds with many health benefits. Natural antioxidants can minimise the risk of chronic illnesses, including cancer, cardiovascular and neurodegenerative diseases (Rezaie et al., 2015). Tocopherols are considered as natural antioxidants and act as free radical scavengers (Poudel et al., 2019). Phenolic compounds have outstanding antimicrobial, antioxidant and anticancer activities (Rafiee et al., 2016; Wambura et al., 2011).

Oil seeds and nuts are good source of natural antioxidants; among these, kernel oil of bene is a rich source of tocopherols and phenolic compounds, which are in high concentration in this plant than reported in common vegetable oils (Farhoosh et al., 2008b).

Phenolic and tocopherol compounds extracted from medicinal plants are the most important bioactive compounds, although their free use in food has its limitations (Fang and Bhandari, 2010), as they may cause undesirable taste. On the other hand, food antioxidants should maintain their activity during storage and remain stable during food processing (Rashidinejad et al., 2016).

Liposomal delivery systems, such as microemulsification and liposomal entrapment at nano- and micro levelss, are performed in food industry to address such challenges (Nedovic et al., 2011; Rafiee et al., 2016). Unfortunately, most of these techniques have weak points such as need of high temperature and the prerequisite of large quantities of emulsifiers and surfactants, which are harmful for human consumption (Rashidinejad et al., 2016). By employing liposomal formulations that require low heat and low amount of surfactants or emulsifiers can overcome all of these shortcomings (Mozafari, 2010). The Mozaffari method, which is simple and easy, is used for preparing liposomes. In this method, liposomes can be prepared in the absence of organic solvents with low shear forces. It is also economical and capable of producing bioactive carriers (Mozafari et al., 2008). Liposomes are usually prepared with high-purity lecithin plus cholesterol, which can be used in trapping, delivering and releasing hydrophilic, adipose-friendly and amphiphilic compounds (dual-friendly) because of its hydrophilic and lipid-like components (Lu et al., 2011).

Many researches have been conducted recently based on liposomes in food science because of the enrichment of bioactive compounds and production of functional foods. Some of these researches are as follows: production of liposome containing vitamins $\mathrm{E}$ and $\mathrm{C}$ with encapsulation efficiency of $86 \%$ and $99 \%$, respectively (Marsanasco et al., 2011). Encapsulation of grape seed polyphenols and anthocyanins of Hibiscus sabdariffa in soy lecithin is reported by using the method of microfluidiser (Gibis et al., 2012, 2014).

Bene trees (Pistacia atlantica) grow in many parts of Iran, and bene oil has high amounts of tochopherol and phenolic compounds, as well as strong antioxidants, and possesses high oxidative stability. The purpose of this study is to produce microliposomes of bene oil with different formulations and to determine their physical stability in order to find the best formulation.

\section{Materials and methods}

Soy lecithin (99\% purity) was prepared from lipoid (GmbH, Ludwigshafen, Germany) as well as cholesterol (95\% purity) from Lifeline (Frederick, MD, USA). In the experiments, all chemicals used were of analytical grade and pharmaceutical standard.

\section{Oil extraction}

Fresh fruits of Pistacia atlantica were acquired from Kerman (Southern Iran). Pharmacology Department of Tehran University authenticated them. For oil extraction, Pistacia fruits were dried in shade, and pericarps of kernels were removed. Dried kernels were subjected to grinding in Cuisinart SG-10 Electric Spice-and-Nut grinder. The dried kernel powder was treated with $n$-hexane $(1: 4 \mathrm{w} / \mathrm{v})$ by agitation in a dark place at ambient temperature for $48 \mathrm{~h}$. Finally, the solvent was evaporated in vacuum at $40^{\circ} \mathrm{C}$ (Rezaie et al., 2015) and nitrogen gas was used to eliminate trace amounts of remaining solvent (Farhoosh et al., 2008a).

\section{Total phenolic (TP) content}

The amount of TP content was evaluated according to the method described by Asnaashari et al. (2015) and the results were expressed in terms of milligram gallic acid equivalents per gram of oil. Folin-Ciocalteu's reagent was used in this method. The calibration curve was plotted and the absorption of bene oil samples was determined at $765 \mathrm{~nm}$ after preparation.

\section{Total tocopherols (TT) content}

The amount of TT content of bene oil samples was characterised according to the method described by Farhoosh et al. (2011) based on $\alpha$-tocopherol. The results were expressed in terms of microgram of $\alpha$-tocopherol equivalents per gram of oil. The calibration curve was plotted and the absorption of oil samples was determined at $520 \mathrm{~nm}$ after preparation. 


\section{Formulation of liposomes}

Carrier materials containing lecithin and cholesterol in the ratios of 60:0, 50:10, 40:20 and 30:30 mg were distilled with 20-mL of purified water for $1 \mathrm{~h}$ at $55^{\circ} \mathrm{C}$, and $5 \mathrm{~mL}$ of bene oil was dissolved in 14-mL polyethylene glycol $3 \% \mathrm{v} / \mathrm{v}$ at $55^{\circ} \mathrm{C}$ for $15 \mathrm{~min}$. The mixture containing bene oil, lecithin and cholesterol was transferred to $300-\mathrm{mL}$ heat-resistant glass beaker and 14-mL polyethylene glycol was added to it. Twin 80 was used as an emulsifier. The mixture was diluted with $40 \mathrm{~mL}$ of distilled water and heated at $55^{\circ} \mathrm{C}$ for $1 \mathrm{~h}$ on a hot plate stirrer at approximately $1,000 \times \mathrm{g}$ (IKA Corporation, Germany). In order to maintain liposomal solution, it was kept at room temperature for $15 \mathrm{~min}$ and stored in refrigerator at $4^{\circ} \mathrm{C}$. (Mozafari, 2010)

In all, the following four treatments were examined:

$\mathrm{T}_{1}$ : lecithin $60 \mathrm{mg}+$ cholesterol $0 \mathrm{mg}+$ bene oil

$\mathrm{T}_{2}$ : lecithin $50 \mathrm{mg}+$ cholesterol $10 \mathrm{mg}+$ bene oil

$\mathrm{T}_{3}$ : lecithin $40 \mathrm{mg}+$ cholesterol $20 \mathrm{mg}+$ bene oil

$\mathrm{T}_{4}$ : lecithin $30 \mathrm{mg}+$ cholesterol $30 \mathrm{mg}$ + bene oil

Physicochemical characterisation of microliposomes were determined as follows.

\section{Encapsulation efficiency (EE)}

The liposomal dispersions were centrifuged at 32,000 $\times \mathrm{g}$ at $4^{\circ} \mathrm{C}$ for $1 \mathrm{~h}$ (Beckman Coulter, USA) to remove free and entrapped bioactive compounds. Then the amounts of total bioactive compounds in initial suspension and supernatant were measured using the LC-MS/MS method. Aliquot of each sample was spiked with internal standard and diluted with acetonitrile. The microliposome encapsulation efficiency (EE) was calculated using the following equation:

$$
E E(\%)=\left[\frac{C_{\text {total }}-C_{\text {free }}}{C_{\text {total }}}\right] \times 100,
$$

where $\mathrm{C}_{\text {total }}$ is the total concentration of bioactive compounds and $\mathrm{C}_{\text {free }}$ is the untrapped bioactive compounds (Poudel et al., 2019).

\section{Particle size, zeta potential and polydispersity index (PDI) measurement}

Particle size, zeta potential and PDI were measured using a dynamic light scattering (DLS) device (Nanotrac Wave, Microtrac, Germany). For this purpose, first each sample was diluted for 50 times using distilled water. Then the samples were transferred to a capillary tube using a syringe; next, the capillary tube was placed at a special place inside the device. Zeta potential of liposomal dispersion was measured at a $\mathrm{pH}$ of 7.4 at $25^{\circ} \mathrm{C}$ and 149 watts (Fatouros and Antimisiaris, 2002).

\section{Evaluation of physical stability}

In order to investigate physical stability, liposomal solutions containing bene oil were kept at $4^{\circ} \mathrm{C}$ for 2 months. Particle size and EE values were carried out to assess physical stability of the samples on days 1, 10, 30 and 60 (Chanda et al., 2011).

\section{Transmission electron microscope (TEM)}

Transmission electron microscope (906 Leo, Zeiss 100 $\mathrm{KV}$, Germany) was used to study the microstructure of nanoliposomes and to confirm their size in nanoscale (less than $100 \mathrm{~nm}$ ). Initially, a drop of optimal microliposome sample was placed on a carbon film grade, and after drying at laboratory temperature, it was imaged by TEM (Klang et al., 2012).

\section{Determination of radical scavenging activity}

Radical scavenging activity of the extract (before and after encapsulation) against 1,1-diphenyl-1-picrylhydrazyl $(\mathrm{DPPH})$ radical was evaluated. First, methanolic extract was prepared in three consecutive dilutions (500, 1,000 and 1,500 ppm); then, $0.1 \mathrm{~mL}$ of each of the extract concentrations was added to 3.9-mL DPPH solution (0.1 mM). After a 30-min incubation period at room temperature, the light absorption of the samples was calculated by spectrophotometer at a wavelength of $517 \mathrm{~nm}$. The percentage of inhibition (I) of free radical DPPH was determined using the following equation:

$$
I(\%)=\frac{A_{\text {blank }}-A_{\text {sample }}}{A_{\text {blank }}} \times 100,
$$

where $A_{\text {blank }}$ is the absorbance of control reaction and $\mathrm{A}_{\text {sample }}$ is the absorbance of test compound (Delazar et al., 2012).

\section{Statistical analysis of data}

The experiments were performed in three replications in a completely randomised design. Statistical analysis of treatments was carried out using analysis of variance (one way ANOVA) by SPSS software version 18.0. Mean significant differences at a level of 0.05 were determined based on Duncan's test. The figures were drawn using Microsoft Excel 2013 software. 


\section{Results and Discussion}

\section{Total phenolic (TP) and total tocopherol s(TT) contents}

Total phenolic content in bene (Pistacia atlantica) oil was 103.47 (mg gallic acid $\mathrm{kg}^{-1}$ of oil) and total TT content was 817.92 (mg a-tocopherol kg-1 of oil). Generally, vegetable oils contain $0.5-2.5 \%$ of phenolic compounds and tocopherols; however, some vegetable oils have extraordinary amounts of up to $5-6 \%$. Bene oil is a rich source of tocopherols as the concentration of TT in bene oil is higher than those reported for some common vegetable oils, for example corn, cottonseed, canola and sunflower have the TT contents of about 605, 630, 640, $695 \mathrm{mg} \mathrm{kg}^{-1}$. Tocopherols, which are considered as rich source of vitamin $\mathrm{E}$, have antioxidant activity and play an important role in prevention and treatment of diseases (Delazar et al., 2012; Farhoosh et al., 2008b). The TP content of olive, corn, sunflower and canola oil is as follows: $15.27,30.80,45.27$ and $18.19 \mathrm{mg}$ gallic acid $\mathrm{kg}^{-1}$ oil, respectively (Farhoosh et al., 2008a). Although the main benefits of phenolic compounds are related mostly to their antioxidant activity, they also offer some important biological activities in vivo and may be beneficial in fighting diseases related to excessive oxygen radical formation in the human body (Farhoosh et al., 2008a).

\section{Physical characteriszation of micro liposomes containing phenolics and tocopherols of liposome size}

Size is an important parameter to assess the stability, liberation of bioactive agents and entrapment efficiency of formulated bioactives (Goli et al., 2005). Table 1 demonstrates particle size of microliposomes with different ratios of lecithin:cholesterol containing $5 \mathrm{mg}$ of phenolic and tocopherol compounds upon a storage time of 60 days. The effect of different ratios of lecithin:cholesterol on the particle size of microliposomes was significant $(P<$ 0.01 ). Table 1 shows that all samples have a micro size of 4-10 $\mu \mathrm{m}$. Comunian et al. (2013) reported an average size of $11.76 \mu \mathrm{m}$ for microcapsules containing vitamin $C$.
According to the results, the mean diameters of liposomes depend on their lecithin composition. Evaluating size of liposomes after 2 months of storage at $4^{\circ} \mathrm{C}$ (Table 1) showed increment in their size. A significant effect was observed in size of liposomes using different ratios of lecithin:cholesterol concentration (Table 1). Microliposomes with a ratio 40:20 $\left(\mathrm{T}_{3}\right)$ had the smallest size.

The results showed that for the liposomal system carrying phenolic and tocopherol compounds of bene oil, there is an optimal phospholipid:cholesterol concentration ratio, which gives the lowest liposomal particle size (Wu et al., 2011).

Contradictory reports are published about the effect of sterols on liposome particle size, which is related to preparation of liposomes. This is in agreement with the report of Gopinath et al. (2004) that increasing the concentration of cholesterol to $30 \%$ reduces the size of vesicles containing azidothymidine. It seems that increase in cholesterol concentration to $30 \%$ damages the structure of liposomes.

\section{Physical stability}

Unchanged parameters, such as particle size, particle size distribution, and encapsulation efficiency, over a long period indicate the stability of produced liposomes. For physical stability, two parameters of particle size and the amount of phenolic and tocopherol residues inside microliposomes during 60 days of storage were used.

In order to evaluate physical stability, the average diameter of liposomal microparticles was studied after storage at $4^{\circ} \mathrm{C}$ for $1,10,30$ and 60 days (Table 1 ). According to the results of analysis of variance, storage time had a significant effect on the size of liposomes $(P<0.05)$ as the size of the microliposomes increased over time. The highest increase was observed in $\mathrm{T}_{1}$ after 60 days. $\mathrm{T}_{3}$ had the smallest increase in size and no significant difference was observed after 60 days. The particle size of $\mathrm{T}_{4}$ also did

Table 1 The particle size (z-average) $(\mu \mathrm{m})$ of liposome containing phenolics and tocopherols during 60 days storage.

\begin{tabular}{|c|c|c|c|c|}
\hline \multirow[t]{2}{*}{ Treatment } & \multicolumn{4}{|c|}{ Storage time (day) } \\
\hline & 1 & 10 & 30 & 60 \\
\hline L 60:C $0^{*}$ & $6.62 \pm 0.03^{\mathrm{Da}}$ & $6.83 \pm 0.22^{\mathrm{Cb}}$ & $7.47 \pm 0.51^{\mathrm{Bab}}$ & $9.25 \pm 0.51^{\mathrm{Aa}}$ \\
\hline L 50:C 10 & $5.43 \pm 0.03^{\mathrm{Cb}}$ & $6.59 \pm 0.21^{\mathrm{Bb}}$ & $7.05 \pm 0.55^{\mathrm{ABb}}$ & $7.45 \pm 0.52^{\mathrm{Ac}}$ \\
\hline L 40:C 20 & $4.39 \pm 0.42^{B C}$ & $4.78 \pm 0.16^{\mathrm{AC}}$ & $4.88 \pm 0.61^{\mathrm{Ac}}$ & $5.04 \pm 0.32^{\mathrm{Ad}}$ \\
\hline L 30:C 30 & $6.24 \pm 0.77^{\mathrm{Cab}}$ & $7.30 \pm 0.79^{\mathrm{Ba}}$ & $7.92 \pm 0.67^{\mathrm{ABa}}$ & $8.44 \pm 0.25^{\mathrm{Ab}}$ \\
\hline
\end{tabular}


not change over 10 to 30 days. The stability of liposomes depends on several factors, including the size and chemical composition of vesicles. Cholesterols increase the density and order of phospholipid molecules because of their location in molecular cavities formed by surfactant monomers, regulate the fluidity of fat membranes, and reduce their permeability to soluble substances. They can also prevent the accumulation of particles during storage by increasing electrostatic repulsion force. In addition, research on the effect of cholesterol on particle stability has shown that cholesterol in optimal amounts increases electrostatic repulsion between particles by increasing membrane stiffness and zeta potential and prevents particles from sticking to each other. However, high amount of cholesterol is likely to cause membrane rupture (Bang et al., 2011).

\section{Zeta potential}

Zeta potential is an indicator of the amount of repulsive interaction between colloidal particles. It is used to assess the stability of a liposomal dispersion. In low-zeta potential particles, there is a small repulsive force, and the particles eventually aggregate and cause system instability. In general, if the total zeta potential of the colloidal system is $\pm 30 \mathrm{mV}$, the particles will be stable in terms of electrostatic repulsive forces (Caddeo et al., 2008; Zou et al., 2014). As illustrated in Table 2, all samples

Table 2 Effects of different ratios of lecithin to cholesterol on Zeta potential.

\begin{tabular}{ll} 
Treatment & Zeta potential $(\mathrm{mV})$ \\
\hline L 60:C $0^{*}$ & $16.4 \pm 0.98^{\mathrm{c}}$ \\
L 50:C 10 & $24.77 \pm 1.53^{\mathrm{b}}$ \\
L 40:C 20 & $30.31 \pm 1.11^{\mathrm{a}}$ \\
L 30:C 30 & $32.39 \pm 0.78^{\mathrm{a}}$
\end{tabular}

*Significant differences in the same column are shown by different letters $(a, b, c)(P<0.05)$. exhibited a negative surface charge in the range of 16.4 to $32.39 \mathrm{mV}$. Regarding zeta potential, $\mathrm{T}_{4}$ had higher absolute charge in all lecithin concentrations and statistically there was no significant difference between $\mathrm{T}_{4}$ and $\mathrm{T}_{3}$ $(P<0.05)$. The zeta potential was increased by increasing the amount of cholesterol in liposomes. The existence of anionic phospholipids (for example phosphatidic acid and phosphatidylserine) and lecithin, and also phenolic compounds, is the reason for negative surface charge of microliposomes. Many researches on liposomes containing phenolic compounds have reported conflicting range of zeta potential values (Hasan et al., 2014).

\section{Polydispersity index (PDI)}

Polydispersity values lower than 0.3 and above, are indicators for homogenous and heterogeneous suspensions, respectively (Hasan et al., 2014). According to Table 3, PDI values of $\mathrm{T}_{3}$ sample were in the range of $0.212-0.308$, which indicated narrow particle size distribution and high homogeneity of produced liposomal system.

\section{Encapsulation efficiency (EE)}

Encapsulation efficiency is the main characteristic of encapsulation proving of loading capacity of a therapeutic agent in liposomes. EE values of microliposomes entrapping tocopherols and phenolic compounds of bene oil in different ratios of lecithin:cholesterol concentrations are given in Table 4. Different ratios of lecithin:cholesterol concentrations had significant effect on EE $(P<0.05)$. EE proportion for all the formulations was in the range of $88.86-55.24 \%$. Storage of samples at $4^{\circ} \mathrm{C}$ for 2 months decreases EE percentage. Increase in EE percentage was observed by increasing lecithin ratio. $\mathrm{T}_{1}$ (60:0) had the highest efficiency. Similarly, Malheiros et al. (2012) reported reduction in EE by addition of cholesterol to liposome containing nisin. According to Liu and Park (2010), the optimal ratio of phospholipid:cholesterol in liposome containing vitamin C was 40:20.

Table 3 Poly dispersity index (PDI) of produced microliposomes during 60 days storage.

\begin{tabular}{|c|c|c|c|c|}
\hline \multirow[t]{2}{*}{ Treatment } & \multicolumn{4}{|c|}{ Storage time (day) } \\
\hline & 1 & 10 & 30 & 60 \\
\hline L 60:C $0^{*}$ & $0.272 \pm 0.05^{\mathrm{Da}}$ & $0.287 \pm 0.01^{\mathrm{Ca}}$ & $0.314 \pm 0.12^{\mathrm{Bc}}$ & $0.563 \pm 0.94^{A}$ \\
\hline L 50:C 10 & $0.260 \pm 0.03^{\mathrm{Ca}}$ & $0.250 \pm 0.07^{\mathrm{Cb}}$ & $0.414 \pm 0.04^{\mathrm{Ba}}$ & $0.518 \pm 0.07^{A B}$ \\
\hline L 40:C 20 & $0.212 \pm 0.16^{C c}$ & $0.221 \pm 0.06^{\mathrm{Cd}}$ & $0.278 \pm 0.09^{B d}$ & $0.308 \pm 0.12^{\mathrm{AC}}$ \\
\hline L 30:C 30 & $0.234 \pm 0.02^{\mathrm{Cb}}$ & $0.236 \pm 0.04^{\mathrm{Cc}}$ & $0.398 \pm 0.10^{\mathrm{Bb}}$ & $0.481 \pm 0.08^{A C}$ \\
\hline
\end{tabular}


Nikkhah $\mathrm{M}$ et al.

Table 4 Encapsulation efficiency of produced microliposomes during 60 days storage.

\begin{tabular}{lcccc} 
Treatment & \multicolumn{4}{c}{ Storage time (day) } \\
\cline { 2 - 5 } & 1 & 10 & 30 & 60 \\
\hline L 60:C 0* & $86.86 \pm 1.90^{\mathrm{Aa}}$ & $77.33 \pm 1.17^{\mathrm{Ba}}$ & $67.26 \pm 1.45^{\mathrm{Ca}}$ & $64.30 \pm 1.38^{\mathrm{Ca}}$ \\
L 50:C 10 & $85.30 \pm 0.04^{\mathrm{Aa}}$ & $71.86 \pm 0.80^{\mathrm{Bb}}$ & $63.33 \pm 0.54^{\mathrm{Cb}}$ & $59.54 \pm 0.58^{\mathrm{Db}}$ \\
L 40:C 20 & $84.33 \pm 0.51^{\mathrm{Aa}}$ & $68.81 \pm 0.11^{\mathrm{Bc}}$ & $59.83 \pm 0.97^{\mathrm{Cc}}$ & $58.49 \pm 1.01^{\mathrm{Cb}}$ \\
L 30:C 30 & $76.80 \pm 0.86^{\mathrm{Ab}}$ & $67.67 \pm 0.37^{\mathrm{Bd}}$ & $59.80 \pm 0.13^{\mathrm{Cc}}$ & $55.24 \pm 0.29^{\mathrm{Dc}}$ \\
\hline
\end{tabular}

*Significant differences in the same column are shown by different letters $(a, b, c)(P<0.05)$.

${ }^{*}$ Significant differences in a same row are shown by different letters $(A, B, C)(P<0.05)$.

Mohammadhasani et al. (2013) concluded that increasing cholesterol in liposome containing gamma oryzanol leads to improvement in EE from 60 to $84 \%$.

Liposomes are mainly composed of phospholipids, and increasing concentration of these improves fluidity of cellular membranes. The addition of cholesterol to the lipid bilayer of liposomes reduces their permeability and increases stability, but hydrophobicity of cholesterol may reduce binding of hydrophilic active substances to liposome surface (Bozzuto and Molinari, 2015).

\section{Morphology and size distribution}

Transmission electron microscope is another perfect technique for obtaining beneficial information regarding shape, particle size and lamellarity of liposomes. The optimal liposome contains phenolic compounds and tocopherols of bene oil, and TEM is the best method to obtain the visual information concerning the morphology and size of liposomes (Hasan et al., 2014). Figure 1 shows liposomes in the micro size range obtained by TEM.

\section{Antioxidant activity}

The antioxidant activity of tocopherols and phenolic compounds of bene oil was determined before and after its encapsulation in liposome in different concentrations using the DPPH method. The liposome showed that the highest percentage of EE $\left(\mathrm{T}_{3}\right)$ was used to investigate the antioxidant activity. Figure 2 shows the amount of DPPH radical scavenging in the presence of different concentrations of capsulated and non-capsulated extracts. Results showed that the concentration of bioactive compound had a significant effect on DPPH free radical scavenging (significant at $P<0.05$ ). The increase in antioxidant activity was proportional to concentration. The highest antioxidant activity observed in tocopherols and phenolic compounds was $1.5 \mathrm{mg} / \mathrm{mL}, 58.2$ and 57.7 respectively. No significant differences were observed in the equal concentrations of capsulated and non-capsulated forms,

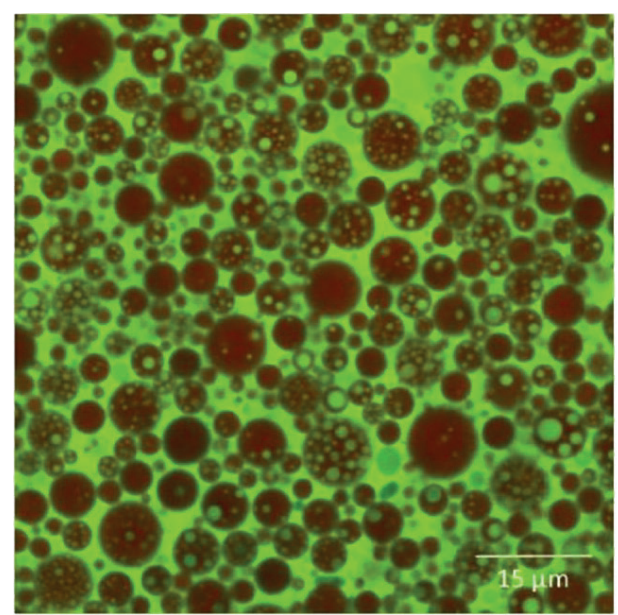

(A) $1 \times$ digital zooming

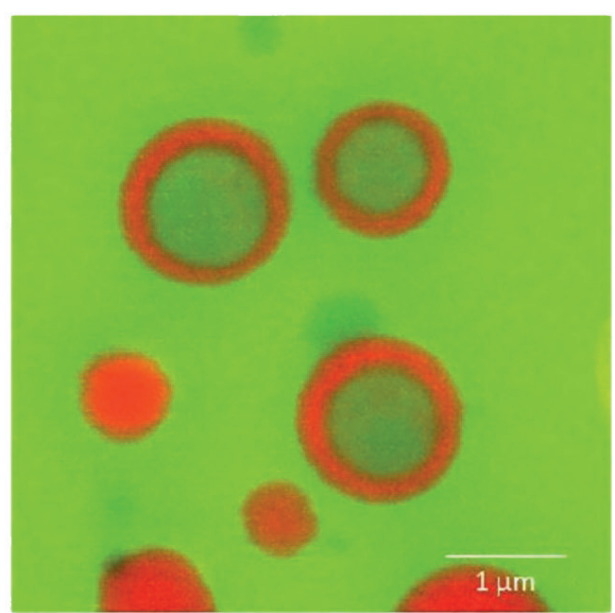

(B) $8 \times$ digital zooming

Figure 1. TEM micrograph of liposome. 


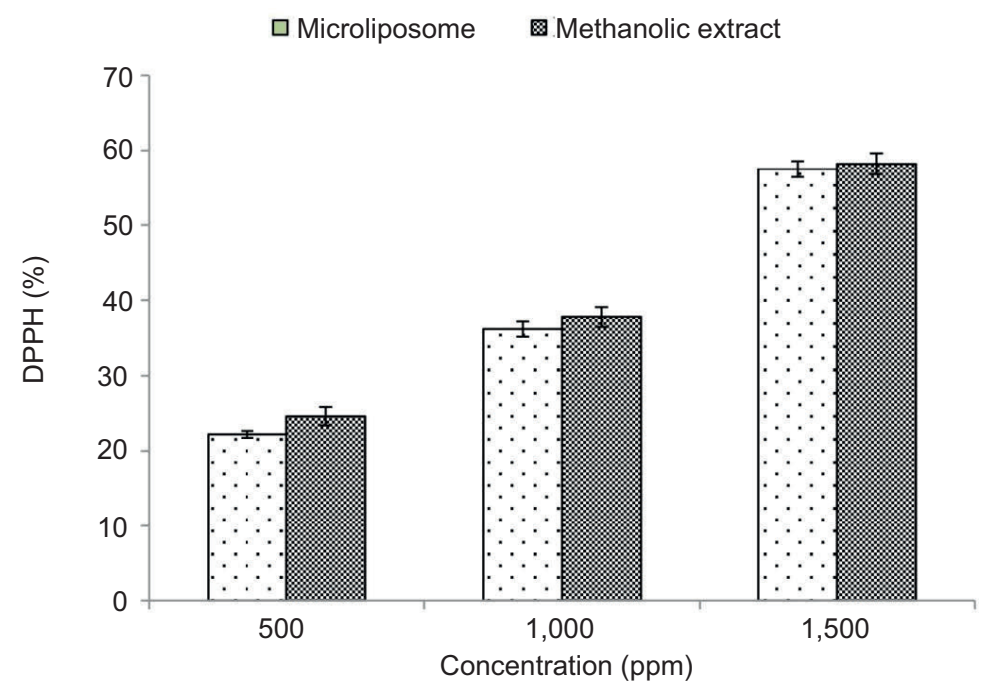

Figure 2. Antioxidant activity of capsulated and non-capsulated liposomes.

which indicate that capsulation had no effect on the antioxidant activity. The results were in agreement with González-Paredes et al. (2011).

\section{Conclusions}

Wild pistachio (pistacia atlantica) as a plant source is rich in phenolic and tocopherol compounds and has significant antioxidant effects. However, the use of phenolic compounds in food products faces numerous limitations because of their low stability, poor solubility, poor bioavailability, interaction with other nutrients, and the creation of chalky taste. Encapsulation could be used as an effective method to improve stability and prevent undesirable taste of phenolic compounds. In the present study, the liposomes were prepared by Mozafari method using different ratios of lecithin:cholesterol (60:0, 50:10, 40:20 and 30:30). Physicochemical characterisation of microliposomes, including characterisation of particle size, zeta potential, EE, size distribution, and morphology, was evaluated. Based on the results, $\mathrm{T}_{3}$ (lecithin:cholesterol ratio of 40:20) was chosen as an optimal formulation because of its higher zeta potential and smaller particle size and suitable stability.

\section{References}

Asnaashari, M., Tajik, R. and Haddad Khodaparast, M.H., 2015. Antioxidant activity of raspberry (Rubusfruticosus) leaves extract and its effect on oxidative stability of sunflower oil. Journal of Food Science and Technology 52(8): 5180-5187. https://doi.org/10.1007/s13197-014-1564-7
Bang, S.H., Hwang, I.C., Yu, Y.M., Kwon, H.R., Kim, D.H. and Park, H.J., 2011. Infuence of chitosan on the liposomal surface on physicochemical properties and the release of nanocarrier system. Journal of Microencapsulation 28(7): 595-640. https:// doi.org/10.3109/02652048.2011.557748

Bozzuto, G. and Molinari, A., 2015. Liposomes as nanomedical devices. International Journal of Nanomedicine 10: 975-999. https://doi.org/10.2147/IJN.S68861

Caddeo, C., Teskac, K., Sinico, C. and Kristl, J., 2008. Effect of resveratrol incorporated in liposomes on proliferation and UV-B protection of cells. International Journal of Pharmaceuticals 36: 183-191. https://doi.org/10.1016/j.ijpharm.2008.07.024

Chanda, H., Das, P., Chakraborty, H. and Ghosh, A., 2011. Development and evaluation of liposomes of fluconazole. Journal of Biomedical and Pharmaceutical Sciences 5: 1-9.

Comunian, T.A., Thomazini, M., Gouvêa Alves, A., Junior, F., Balieiro, J. andFavaro-Trindade, C., 2013. Microencapsulation of ascorbic acid by complex coacervation: protection and controlled release. Food Research International 52: 373-379. https://doi.org/10.1016/j.foodres.2013.03.028

Delazar, A., Lotfipour, F. and Nazemiyeh, H., 2012. Antioxidant and antimicrobial activity of pedicularissibthorpii boiss. And pediculariswilhelmsiana Fisch ex. Advanced Pharmaceutical Bulletin 2(1): 89-92.

Fang, Z. and Bhandari, B., 2010. Encapsulation of polyphenols-a review. Trends in Food Science and Technology 21: 510-523. https://doi.org/10.1016/j.tifs.2010.08.003

Farhoosh, R., Niazmand, R., Rezaei, M. and Sarabi, M., 2008 a. Kinetic parameter determination of vegetable oil oxidation under Rancimat test conditions. European Journal of Lipid Science and Technology 110:587-592. https://doi.org/10.1002/ejlt. 200800004

Farhoosh, R., Tavakoli, J. and Haddad Khodaparast, M.H., 2008ba. Chemical composition and oxidative stability of kernel oils from 
two current subspecies of Pistacia atlantica in Iran. Journal of the American Oil Chemists' Society 85: 723-729. https://doi. org/10.1007/s1174.6-008-1258-2

Farhoosh, R., Tavassoli-Kafrani, MH., Sharif, A., 2011. Antioxidant activity of the fractions separated from the unsaponifiable matter of bene hull oil. Food Chemistry 126(2): https://doi. org/10.1016/j.foodchem.2010.11.047

Fatouros D.G., Antimisiaris S.G., 2002. Effect of amphiphilic drugs on the stability and zeta-potential of their liposome formulations: a study with prednisolone, diazepam, and griseofulvin. Journal of Colloid and Interface Science 251(2): 271-277. https:// doi.org/10.1006/jcis.2002.8432

Gibis, M., Vogt, E. and Weiss, J., 2012. Encapsulation of polyphenolic grape seed extract in polymer-coated liposomes. Food and Function 3: 246-254. https://doi.org/10.1039/C1FO10181A

Gibis, M., Zeeb, B. and Weiss, J., 2014. Formation, characterization, and stability of encapsulated hibiscus extract in multilayered liposomes. Food Hydrocolloids 38: 28-39. https://doi. org/10.1016/j.foodhyd.2013.11.014

Goli, A.H., Barzegar, M. and Sahari, M.A., 2005. Antioxidant activity and total phenolic compounds of pistachio (Pistachiavera) hull extracts. Food Chemistry 92: 521-525. https://doi.org/10.1016/j. foodchem.2004.08.020

González-Paredes, A., Clarés-Naveros, B., RuizMartínez, M.A., Durbán-Fornieles, J.J., Ramos-Cormenzana, A. and MonteolivaSánchez, M., 2011. Delivery systems for natural antioxidant compounds: archaeosomes and archaeosomal hydrogels characterization and release study. International Journal of Pharmaceutics 421(2): 321-331. https://doi.org/10.1016/j. ijpharm.2011.09.042

Gopinath, D., Ravi, D., Rao, B., Apte, S., Renuka, D. and Rambhau, D.,2004. Ascorbyl palmitate vesicles (Aspasomes): formation, characterization and applications. International Journal of Pharmaceutics 271(1): 95-113. https://doi.org/10.1016/j. ijpharm.2003.10.032

Hasan, M., Belhaj, N., Benachour, H., Barberi-Heyob, M., Kahn, C.J.F., Jabbari, E. and Arab-Tehrany, E., 2014. Liposome encapsulation of curcumin: physicochemical characterizations and effects on MCF7 cancer cell proliferation. International Journal of Harmaceutics 461: 519-528. https://doi.org/10.1016/j. ijpharm.2013.12.007

Klang, V., Matsko, N.B., Valenta, C. and Hofer, F., 2012. Electron microscopy of nanoemulsions: an essential tool for characterization and stability assessment. Micron 43: 85-103. https://doi. org/10.1016/j.micron.2011.07.014

Liu, N. and Park, H.J., 2010. Factors effect on the loading efficiency of vitamin $\mathrm{C}$ loaded chitosan-coated nanoliposomes. Colloids and Surfaces B. Biointerfaces 76: 16-19. https://doi. org/10.1016/j.colsurfb.2009.09.041

Lu, Q., Li, D. and Jikng, J., 2011. Preparation of a tea polyphenol nanoliposome system and its physicochemical properties. Journal of Agricultural and Food Chemistry 59: 13004-13011. https://doi. org/10.1021/jf203194w

Malheiros, P.D.S., Sant Anna, V., Barbosa, M.D.S., Brandelli, A. and Franco, B.D.G.D.M.,2012. Effect of liposome-encapsulated nisin and bacteriocin-like substance P34 on Listeria monocytogenes growth in Minas technological approach. Biotechnology Annual Review 7: 59-85. https://doi.org/10.1016/j. ijfoodmicro.2012.04.004

Marsanasco, M., Márquez, A.L., Wagner, J.R., Alonso, S.D.V. and Chiaramoni, N.S., 2011. Liposomes as vehicles for vitamins E and $\mathrm{C}$ : an alternative to fortify orange juice and offer vitamin C protection after heat treatment. Food Research International 44(9): 3039-3046. https://doi.org/10.1016/j.foodres.2011.07.025

Mohammadhasani, M., Ghanbarzadeh, B., Hamishehkar, H., RezaeiMokarram, R. and Mohammadifar, M.A., 2013. Gamma oryzanol bearing nanoliposome producedly by modified thermal method: thermal property, encapsulation efficiency, oscillatory rheometry. Iranian Journal of Ploymer Science and Technology 26(5): 413-425.

Mozafari, M.R., 2010. Nanoliposomes: preparation and analysis. Methods in Molecular Biology (Clifton, N.J.) 605: 29-50. https://doi.org/10.1007/978-1-60327-360-2_2

Mozafari, M.R., Johnson, C., Hotziantoniou, S. and Demetzos, C., 2008. Nanoliposomes and their applications in food nanotechnology. Journal of Liposome Research18: 309-327.

Nedovic, V., Kalusevic, A., Manojlovic, V., Levic, S. and Bugarski, B., 2011. An overview of encapsulation technologies for food applications. Procedia Food Science 1: 1806-1815. https://doi. org/10.1016/j.profoo.2011.09.265

Poudel, A., Gachumi, G., Wasan, K., DallalBashi, Z., El-Aneed, A. and Badea, I., 2019. Development and characterization of liposomal formulations containing phytosterols extracted from canola oil deodorizer distillate along with tocopherols as food additives. Pharmaceutics11: 185-198. https://doi.org/10.3390/ pharmaceutics11040185

Rafiee, Z., Barzegar,M., Sahari, M.A. and Maherani,M., 2016. Nanoliposomal carriers for improvement the bioavailability of high-valued phenolic compounds of pistachio green hull extract. Food Chemistry 220: 115-122. https://doi.org/10.1016/j. foodchem.2016.09.207

Rashidinejad, A., Birch, E.J., Sun-Waterhouse, D. and Everett, D.W., 2016. Effect of liposomal encapsulation on the recovery and antioxidant properties of green tea catechins incorporated into a hard low-fat cheese following in vitro simulated gastrointestinal digestion. Food and Bioproducts Processing. 100: 238-245. https://doi.org/10.1016/j.fbp.2016.07.005

Rezaie, M., Farhoosh, R., Iranshahi, M., Sharif,A. and Golmohamadzadeh, S., 2015. Ultrasonic-assisted extraction of antioxidative compounds from bene (Pistacia atlantica subsp. mutica) hull using various solvents of different physicochemical properties. Food Chemistry 173: 577-583. https://doi. org/10.1016/j.foodchem.2014.10.081

Sharif, A., Farhoosh,R., Haddad Khodaparast, A.H. and Tavassoli Kafrani, M.H., 2009. Antioxidant activity of Bene hull oil compared with sesame and rice bran oils during the frying process of sunflower. Journal of Food Lipids 16: 394-406. https://doi. org/10.1111/j.1745-4522.2009.01154.x

Wambura, P., Yang, W. and Mwakatage, N.R., 2011. Effects of sonication and edible coating containing rosemary and tea extracts 
on reduction of peanut lipid oxidative rancidity. Food and Bioprocess Technology 4: 107-115. https://doi.org/10.1007/ s11947-008-0150-2

Wong, M.L., Timms, R.E. and Goh, E.M.,1988. Colorimetric determination of total tocopherols in palm oil, olein and stearin. Journal of the American Oil Chemists' Society 65: 258-261. https://doi.org/10.1007/BF02636412

Wu, L., Zhang, J. and Watanabe, W., 2011. Physical and chemical stability of drug nanoparticle. Advanced Drug Delivery Reviews 63(6): 456-469. https://doi.org/10.1016/j.addr.2011.02.001
Zou, L.Q., Liu, W., Liu, W.L., Liang, R.H., Li, T., Liu, C.M., Cao, Y.L., Niu, J. and Liu, Z., 2014. Characterization and bioavailability of tea polyphenol nanoliposome prepared by combining an ethanol injection method with dynamic high-pressure microfluidization. Journal of Agricultural and Food Chemistry 62: 934-941. https://doi.org/10.1021/jf402886s 